\title{
BCK is not Structurally Complete
}

\author{
Tomasz Kowalski
}

\begin{abstract}
We exhibit a simple inference rule, which is admissible but not derivable in $\mathrm{BCK}$, proving that $\mathrm{BCK}$ is not structurally complete. The argument is proof-theoretical.
\end{abstract}

\section{Introduction}

By a logic we mean a set of formulae closed under a consequence operation given by some axioms and structural inference rules. An inference rule of the form

$$
\frac{\alpha_{1}, \ldots, \alpha_{n}}{\beta}
$$

is derivable in a logic $L$ if $\beta \in C\left(\left\{\alpha_{1}, \ldots, \alpha_{n}\right\}\right)$, where $C$ is the consequence operation of $L$. An inference rule is admissible if $\sigma\left(\alpha_{1}\right), \ldots, \sigma\left(\alpha_{n}\right) \in L$ implies $\sigma(\beta) \in L$ for every substitution $\sigma$. Admissibility is often informally expressed by saying that adjoining an admissible rule to the consequence operation of $L$ does not change the set of theorems. A logic $L$ is structurally complete if every rule admissible in $L$ is also derivable in $L$.

The notion of structural completeness was isolated by Pogorzelski in [7], and after a few decades of dormancy is undergoing a revival. For example, Olson, Raftery, and van Alten [6] deal with substructural logics without contraction, and Cintula and Metcalfe [2] investigate structural completeness in fuzzy logics. A number of general results have been proved and the question of structural completeness settled for many important logics. A notable exception to this is BCK, a logic of pure implication and one of the senior members of the family of substructural logics. BCK, whose name derives from a connection to combinators $B, C$, and $K$ (see e.g., Curry, Hindley, and Seldin [3]), is typically defined by the following axioms:

(B) $(\varphi \rightarrow \psi) \rightarrow((\chi \rightarrow \varphi) \rightarrow(\chi \rightarrow \psi))$,

(C) $(\varphi \rightarrow(\psi \rightarrow \chi)) \rightarrow(\psi \rightarrow(\varphi \rightarrow \chi))$,

(K) $\varphi \rightarrow(\psi \rightarrow \varphi)$,

Received March 25, 2012; accepted September 30, 2012

2010 Mathematics Subject Classification: Primary 03B47; Secondary 06F35, 03 F07

Keywords: BCK logic, structural completeness, admissible rules

(C) 2014 by University of Notre Dame 10.1215/00294527-2420642 
and the sole inference rule

$$
\frac{\varphi, \varphi \rightarrow \psi}{\psi}
$$

of modus ponens. Observe that $(\alpha \rightarrow \alpha) \rightarrow(\beta \rightarrow \beta)$ is a theorem of $\mathrm{BCK}$, so the truth constant 1 is definable, for example, by putting $1=x \rightarrow x$ for a distinguished variable $x$. The usual algebraic semantics for BCK, introduced in Iseki [4], is the quasivariety of $B C K$-algebras, with the following base of quasi-identities:

1. $x \rightarrow 1=1$,

2. $1 \rightarrow x=x$

3. $(x \rightarrow y) \rightarrow((y \rightarrow z) \rightarrow(x \rightarrow z))=1$,

4. $x \rightarrow(y \rightarrow z)=y \rightarrow(x \rightarrow z)$,

5. $x \rightarrow y=1=y \rightarrow x$ implies $x=y$.

The quasivariety of BCK-algebras forms an equivalent quasivariety semantics (see Blok and Pigozzi [1]) for BCK. The question of structural completeness of BCK was mentioned as open in [6].

\section{Gentzen Calculus for BCK}

From a proof-theoretical perspective, a particularly handy presentation of BCK employs a Gentzen-style sequent system. Sequents are pairs $\Gamma \Rightarrow \alpha$, with $\Gamma$ a multiset of terms, $\alpha$ a term, and $\Rightarrow$ the separator. Terms are formulae of BCK. Out of several versions of sequent calculus for BCK, we choose the following: initial sequents

$$
\Gamma, x \Rightarrow x
$$

where $x$ is a variable, and logical inference rules

$$
\frac{\Gamma \Rightarrow \alpha \quad \Delta, \beta \Rightarrow \gamma}{\Gamma, \Delta, \alpha \rightarrow \beta \Rightarrow \gamma}(\rightarrow \Rightarrow), \quad \frac{\Gamma, \alpha \Rightarrow \beta}{\Gamma \Rightarrow \alpha \rightarrow \beta}(\Rightarrow \rightarrow) .
$$

It can be shown by induction on complexity of $\alpha$ that the sequent $\Gamma, \alpha \Rightarrow \alpha$ is provable for any multiset of terms $\Gamma$ and any term $\alpha$. Structural rules of cut and weakening

$$
\frac{\Gamma \Rightarrow \alpha \quad \Delta, \alpha \Rightarrow \beta}{\Gamma, \Delta \Rightarrow \beta}, \quad \frac{\Gamma \Rightarrow \alpha}{\beta, \Gamma \Rightarrow \alpha}
$$

are not in the official stock of rules. The next lemma justifies this choice.

Lemma 2.1 Cut and weakening are eliminable in $B C K$. The rule $(\Rightarrow \rightarrow)$ is invertible in $B C K$.

Eliminability here means that whenever the upper sequents are provable, the lower sequent is provable as well. Invertibility means that swapping the lower and upper sequents in $(\Rightarrow \rightarrow)$ results in an eliminable rule. It follows that a sequent $\Gamma \Rightarrow \alpha$ is provable if and only if the sequent $\Gamma, \alpha_{n}, \ldots, \alpha_{1} \Rightarrow \alpha_{0}$ is provable, where

$$
\alpha=\alpha_{n} \rightarrow\left(\alpha_{n-1} \rightarrow \cdots \rightarrow\left(\alpha_{1} \rightarrow \alpha_{0}\right) \cdots\right)
$$

and $\alpha_{0}$ is a variable or the constant 1 . Clearly, each BCK term $\alpha$ is of the above form. We will use a shorthand notation $\alpha_{1} \alpha_{2} \cdots \alpha_{n} \rightarrow \alpha_{0}$, where the antecedent is thought of as a multiset of terms. For a multiset $\Gamma$ of terms and a term $\varphi$ we will simply write $\Gamma \rightarrow \varphi$, and, by extension, for multisets $\Gamma$ and $\Delta$ we will write $\Gamma \Delta \rightarrow \varphi$ for $\Delta \rightarrow(\Gamma \rightarrow \varphi)$. 
The next lemma states three further properties of a Gentzen-style system for BCK that will be used later without notice. All three are well known to hold in BCK, and can be easily proved using Lemma 2.1, so we leave the proofs to the reader.

Lemma 2.2 Let $\Gamma, \Delta$ be multisets of terms, and let $\alpha, \beta$ be terms. Then the following hold.

1. The sequent $\Gamma \rightarrow \alpha, \Gamma \Rightarrow \alpha$ is provable.

2. If $\Gamma \subseteq \Delta$, then the sequent $\Gamma \rightarrow \alpha \Rightarrow \Delta \rightarrow \alpha$ is provable.

3. If $\beta$ is a theorem, then the sequent $\beta \Gamma \rightarrow \alpha \Rightarrow \Gamma \rightarrow \alpha$ is provable.

\section{Split Terms in BCK}

Split terms can be thought of as encoding cut-free proofs of provable sequents in which they occur. The notion was introduced by the author in [5] for the logic BCI, a close relative of BCK. In the first part of this section, we define split terms for BCK. In the second part, we prove two lemmas whose import is that some special provable sequents can be simplified by means of split terms.

Lemma 3.1 The sequent $\Gamma \Rightarrow v$, where $v$ is a variable and $v \notin \Gamma$, is provable if and only if there exist $a \gamma=\gamma_{n} \gamma_{n-1} \cdots \gamma_{1} \rightarrow \gamma_{0} \in \Gamma$ and a partition $\left\{\Gamma_{i}\right\}_{i=1}^{n}$ of $\Gamma \backslash\{\gamma\}$ such that

1. $\gamma_{0}=v$,

2. for every $i \in\{1, \ldots, n\}$ there is a $\Gamma_{i}$ such that $\Gamma_{i} \Rightarrow \gamma_{i}$ is provable.

Proof Notice that only the forward direction is nontrivial. We proceed by induction on the length of the cut-free proof of $\Gamma \Rightarrow v$. If this is an initial sequent, the lemma holds vacuously. For the inductive step, the last rule in a cut-free proof of $\Gamma \Rightarrow v$ must be

$$
\frac{\Pi \Rightarrow \alpha \quad \Delta, \beta \Rightarrow v}{\Gamma \Rightarrow v}
$$

with $\alpha \rightarrow \beta \in \Gamma$ and $\Pi, \Delta=\Gamma \backslash\{\alpha \rightarrow \beta\}$. Notice that $v \notin \Delta$, because otherwise $\Gamma \Rightarrow v$ would be an initial sequent. Arguing case by case, we will show that the requirements of the lemma are satisfied. (1) If $\Delta, \beta \Rightarrow v$ is an initial sequent, then $\beta=v$ and so $\Gamma=\Pi, \Delta, \alpha \rightarrow v$. Since $\Pi \Rightarrow \alpha$ is provable, by weakening we get $\Pi, \Delta \Rightarrow \alpha$ and thus $\alpha \rightarrow \beta$ satisfies the requirements. (2) If $\Delta, \beta \Rightarrow v$ is not initial, the inductive hypothesis applies to $\Delta, \beta \Rightarrow v$ and thus there is a term $\delta=\delta_{n} \delta_{n-1} \cdots \delta_{1} \rightarrow v$ and a partition of $(\Delta \cup\{\beta\}) \backslash\{\delta\}$ into $\Delta_{1}, \ldots, \Delta_{n}$ such that the sequents $\Delta_{i} \Rightarrow \delta_{i}$ are provable. Now there are two further cases. (2.1) If $\beta=\delta$, then $\alpha \rightarrow \beta$ satisfies all requirements of the lemma. (2.2) If $\beta \neq \delta$ and $\beta \in \Delta_{j}$ for some $j \in\{1, \ldots, n\}$, then the sequent $\Pi, \Delta_{j} \backslash \beta, \alpha \rightarrow \beta \Rightarrow \delta_{j}$ is provable by application of

$$
\frac{\Pi \Rightarrow \alpha \quad \Delta_{j} \Rightarrow \delta_{j}}{\Pi, \Delta_{j} \backslash\{\beta\}, \alpha \rightarrow \beta \Rightarrow \delta_{j}} .
$$

Then, we obtain provable sequents

$$
\begin{aligned}
\Delta_{1} & \Rightarrow \delta_{1} \\
\vdots & \vdots \\
\Delta_{j-1} & \Rightarrow \delta_{j-1} \\
\Pi, \Delta_{j} \backslash\{\beta\}, \alpha \rightarrow \beta & \Rightarrow \delta_{j}
\end{aligned}
$$




$$
\begin{gathered}
\Delta_{j+1} \Rightarrow \delta_{j+1} \\
\vdots \quad \vdots \\
\Delta_{n} \Rightarrow \delta_{n},
\end{gathered}
$$

where

$$
\Delta_{1}, \ldots, \Delta_{j-1}, \Pi, \Delta_{j} \backslash\{\beta\}, \alpha \rightarrow \beta, \Delta_{j+1}, \ldots, \Delta_{n}=\Gamma \backslash\{\delta\}
$$

and the requirements of the lemma are satisfied by $\delta$. This ends the proof.

For a provable sequent $\Gamma \Rightarrow v$, any term $\gamma$ satisfying the lemma above will be called a split term. Such a $\gamma$ may not be unique, and so for a given sequent $\Gamma \Rightarrow v$ different cases of split terms often need to be considered. To indicate a particular split term $\gamma$, we will say that $\Gamma \Rightarrow v$ is provable with split term $\gamma$, or that $\gamma$ splits in $\Gamma \Rightarrow v$.

Now we move on to the simplification lemmas mentioned at the beginning of the section. They deal with two different cases of a split term in a provable sequent of the form $\Gamma, \alpha \Gamma \rightarrow x \Rightarrow x$.

Lemma 3.2 Let $\Gamma=\left\{\gamma_{1}, \ldots, \gamma_{n}\right\}$ be a multiset of terms. Suppose that the sequent $\Gamma, \alpha \Gamma \rightarrow x \Rightarrow x$ is provable with split term $\gamma_{1}=\beta_{1} \cdots \beta_{k} \rightarrow x$. Then, for some $\Gamma^{\prime} \subseteq \Gamma \backslash\left\{\gamma_{1}\right\}$ and some $i \in\{1, \ldots, n\}$, the sequent $\alpha \Gamma^{\prime} \rightarrow \beta_{i} \Rightarrow \Gamma^{\prime} \rightarrow \beta_{i}$ is provable. Moreover, the sequent $\Gamma^{\prime} \rightarrow \beta_{i} \Rightarrow \Gamma \rightarrow x$ is also provable.

Proof Since $\gamma_{1}=\beta_{1} \cdots \beta_{k} \rightarrow x$ is split, after a harmless renumbering of $\gamma_{2}, \ldots, \gamma_{n}$ we get the following provable sequents:

$$
\begin{aligned}
\gamma_{2}, \ldots, \gamma_{p}, \alpha \Gamma \rightarrow x & \Rightarrow \beta_{1} \\
\Lambda_{2} & \Rightarrow \beta_{2} \\
\vdots & \vdots \\
\Lambda_{k} & \Rightarrow \beta_{k},
\end{aligned}
$$

where $\Lambda_{2}, \ldots, \Lambda_{k}=\gamma_{p+1}, \ldots, \gamma_{n}$ and $2 \leq p \leq n$. Writing $\Gamma$ in full, we have

$$
\gamma_{2}, \ldots, \gamma_{p},\left(\beta_{1} \cdots \beta_{k} \rightarrow x\right) \rightarrow\left(\alpha \gamma_{2} \cdots \gamma_{n} \rightarrow x\right) \Rightarrow \beta_{1} \text {; }
$$

therefore,

$$
\left(\beta_{1} \cdots \beta_{k} \rightarrow x\right) \rightarrow\left(\alpha \gamma_{2} \cdots \gamma_{n} \rightarrow x\right) \Rightarrow \gamma_{2} \cdots \gamma_{p} \rightarrow \beta_{1}
$$

is provable. We will now show that

$$
\alpha \gamma_{2} \cdots \gamma_{p} \rightarrow \beta_{1} \Rightarrow\left(\beta_{1} \cdots \beta_{k} \rightarrow x\right) \rightarrow\left(\alpha \gamma_{2} \cdots \gamma_{n} \rightarrow x\right)
$$

is also provable. This is the case if and only if the sequent

$$
\alpha, \gamma_{2}, \ldots, \gamma_{n}, \beta_{1} \cdots \beta_{k} \rightarrow x, \alpha \gamma_{2} \cdots \gamma_{p} \rightarrow \beta_{1} \Rightarrow x
$$

is provable. Now, consider the following system of sequents:

$$
\begin{aligned}
\alpha, \gamma_{2}, \ldots, \gamma_{p}, \alpha \gamma_{2} \cdots \gamma_{p} \rightarrow \beta_{1} & \Rightarrow \beta_{1} \\
\Lambda_{2} & \Rightarrow \beta_{2} \\
\vdots & \vdots \\
\Lambda_{k} & \Rightarrow \beta_{k},
\end{aligned}
$$


where $\Lambda_{1}, \ldots, \Lambda_{k}$ are as before. Since all these are clearly provable, we get that (3) is provable with split term $\gamma_{1}=\beta_{1} \cdots \beta_{k} \rightarrow x$, and so (2) is provable. Applying cut to (2) and (1) we obtain

$$
\alpha \gamma_{2} \cdots \gamma_{p} \rightarrow \beta_{1} \Rightarrow \gamma_{2} \cdots \gamma_{p} \rightarrow \beta_{1},
$$

which yields the desired conclusion, with $\Gamma^{\prime}=\left\{\gamma_{2}, \ldots, \gamma_{p}\right\}$. A parallel argument shows that the sequent

$$
\gamma_{1}, \ldots, \gamma_{n}, \gamma_{2} \cdots \gamma_{p} \rightarrow \beta_{1} \Rightarrow x
$$

is provable with split term $\gamma_{1}$. This proves the "moreover" part.

Lemma 3.3 Let $\Gamma=\left\{\gamma_{1}, \ldots, \gamma_{n}\right\}$ be a multiset of terms. Suppose that the sequent $\Gamma, \alpha \Gamma \rightarrow x \Rightarrow x$ is provable with split term $\alpha \Gamma \rightarrow x \Rightarrow x$ and that $\alpha$ is not a theorem. Then, some $\gamma \in \Gamma$ is a theorem. Therefore, the sequent $\Gamma^{\prime}, \alpha \Gamma^{\prime} \rightarrow x \Rightarrow x$ is provable, where $\Gamma^{\prime}=\Gamma \backslash\{\gamma\}$.

Proof Since $\alpha \Gamma \rightarrow x$ is split, we have

$$
\begin{gathered}
\Lambda_{1} \Rightarrow \alpha \\
\Lambda_{2} \Rightarrow \gamma_{1} \\
\vdots \quad \vdots \\
\Lambda_{n+1} \Rightarrow \gamma_{n}
\end{gathered}
$$

with $\Lambda_{1}, \ldots, \Lambda_{n+1}=\gamma_{1}, \ldots, \gamma_{n}$, so at least one $\Lambda_{i}$ must be empty. As $\alpha$ is not a theorem, $i \in\{2, \ldots, n+1\}$, and so $\gamma_{i-1}$ is a theorem. Thus, we have the following system of provable sequents:

$$
\begin{aligned}
\Lambda_{1} & \Rightarrow \alpha \\
\Lambda_{2} & \Rightarrow \gamma_{1} \\
\vdots & \vdots \\
\Lambda_{i-1} & \Rightarrow \gamma_{i-2} \\
& \Rightarrow \gamma_{i-1} \\
\Lambda_{i+1} & \Rightarrow \gamma_{i} \\
\vdots & \vdots \\
\Lambda_{n+1} & \Rightarrow \gamma_{n} .
\end{aligned}
$$

Putting $\gamma=\gamma_{i-1}$, we get that $\gamma$ is a theorem, as required. Consider the sequent $\alpha \Gamma \rightarrow x \Rightarrow \alpha \Gamma^{\prime} \rightarrow x$. Since $\Gamma^{\prime}=\Gamma \backslash\{\gamma\} \subseteq \Gamma$, we get that $\alpha \Gamma^{\prime} \rightarrow x \Rightarrow \alpha \Gamma \rightarrow x$ is provable. As the sequent $\Gamma, \alpha \Gamma \rightarrow x \Rightarrow x$ is provable by assumption, we apply cut

$$
\frac{\alpha \Gamma^{\prime} \rightarrow x \Rightarrow \alpha \Gamma \rightarrow x \quad \Gamma, \alpha \Gamma \rightarrow x \Rightarrow x}{\Gamma, \alpha \Gamma^{\prime} \rightarrow x \Rightarrow x}
$$

and obtain that $\gamma, \Gamma^{\prime}, \alpha \Gamma^{\prime} \rightarrow x \Rightarrow x$ is provable. But $\gamma$ is provable, so another application of cut

$$
\frac{\Rightarrow \quad \gamma, \Gamma^{\prime}, \alpha \Gamma^{\prime} \rightarrow x \Rightarrow x}{\Gamma^{\prime}, \alpha \Gamma^{\prime} \rightarrow x \Rightarrow x}
$$

shows that the sequent $\Gamma^{\prime}, \alpha \Gamma^{\prime} \rightarrow x \Rightarrow x$ is provable, as claimed. 


\section{Structural Incompleteness}

We now have all the tools we need to prove structural incompleteness of BCK. First we will prove an auxiliary result that amounts to admissibility of a nonstandard, disjunctive inference rule.

Lemma 4.1 Suppose that the sequent $\alpha \rightarrow \beta \Rightarrow \beta$ is provable. Then $\alpha$ is a theorem or $\beta$ is a theorem.

Proof Induction on complexity of $\alpha \rightarrow \beta$ measured by the number of occurrences of the connective. If there is a single occurrence, $\alpha$ is a variable or the constant 1 and so is $\beta$. If any of them is 1 , the claim holds, and if both are variables, $\alpha \rightarrow \beta \Rightarrow \beta$ is not provable, so the claim holds as well. For the inductive step, we proceed by contradiction. Suppose that there are $\alpha$ and $\beta$ for which the claim fails, and that $\alpha \rightarrow \beta$ is the shortest such counterexample. Now, $\beta$ is $\Gamma \rightarrow x$ for some multiset $\Gamma$ of terms and a variable $x$. Consider the sequent $\Gamma, \alpha \Gamma \rightarrow x \Rightarrow x$. By assumption it is provable, so either it is initial or it has a split term. If it is initial, then $x \in \Gamma$, but then $\beta=\Gamma \rightarrow x$ is a theorem, contradicting the assumption. So $\Gamma, \alpha \Gamma \rightarrow x \Rightarrow x$ must have a split term, and we have two cases to consider.

Case 1. If $\alpha \Gamma \rightarrow x \Rightarrow x$ is split, then, since $\alpha$ is not a theorem, Lemma 3.3 applies and $\Gamma^{\prime}, \alpha \Gamma^{\prime} \rightarrow x \Rightarrow x$ is provable. Putting $\beta^{\prime}=\Gamma^{\prime} \rightarrow x$, we obtain that $\alpha \rightarrow \beta^{\prime} \Rightarrow \beta^{\prime}$ is provable, and $\alpha \rightarrow \beta^{\prime}$ is shorter than $\alpha \rightarrow \beta$. Thus, by inductive hypothesis, $\alpha$ or $\beta^{\prime}$ is a theorem. But $\alpha$ is not a theorem, therefore $\beta^{\prime}$ is. Now $\beta^{\prime}=\Gamma^{\prime} \rightarrow x$ and $\Gamma^{\prime} \subset \Gamma$, so $\Gamma^{\prime} \rightarrow x \Rightarrow \Gamma \rightarrow x$ is provable and thus $\Gamma \rightarrow x=\beta$ is a theorem. This is a contradiction.

Case 2. If some $\gamma \in \Gamma$ is split, then Lemma 3.2 applies, and we obtain a provable sequent $\alpha \Gamma^{\prime} \rightarrow \beta_{i} \Rightarrow \Gamma^{\prime} \rightarrow \beta_{i}$, where $\Gamma^{\prime} \subseteq \Gamma \backslash\{\gamma\}$ and $\gamma=\beta_{1} \cdots \beta_{k} \rightarrow x$. As in the previous case, $\alpha$ is not a theorem, so by inductive hypothesis we get that $\Gamma^{\prime} \rightarrow \beta_{i}$ is. Then, by the "moreover" part of Lemma 3.2 we get that $\Gamma \rightarrow x=\beta$ is a theorem, which is a contradiction.

Theorem 4.2 The inference rule

$$
\frac{(\alpha \rightarrow \beta) \rightarrow \beta}{(\beta \rightarrow \alpha) \rightarrow \alpha}
$$

is admissible, but not derivable in BCK. Therefore BCK is not structurally complete.

Proof To show admissibility, suppose that $(\alpha \rightarrow \beta) \rightarrow \beta$ is a theorem. Thus, the sequent $(\alpha \rightarrow \beta) \Rightarrow \beta$ is provable. By Lemma 4.1 then, $\alpha$ is a theorem or $\beta$ is a theorem. In either case, $(\beta \rightarrow \alpha) \rightarrow \alpha$ is a theorem.

To prove nonderivability, consider the $\{\rightarrow, 1\}$-reduct of the totally ordered threeelement Heyting algebra $\{0, a, 1\}$. Taking $\alpha$ and $\beta$ to be variables, we define a valuation $v$ putting $v(\alpha)=a$ and $v(\beta)=0$. Then, $v(\alpha \rightarrow \beta)=0$ and so $v((\alpha \rightarrow \beta) \rightarrow \beta)=1$. But $v(\beta \rightarrow \alpha)=1$ and therefore of $v((\beta \rightarrow \alpha) \rightarrow \alpha)=0$.

\section{An Algebraic Corollary}

Admissible rules have a natural algebraic interpretation. Namely, a rule

$$
\frac{\alpha_{1}, \ldots, \alpha_{n}}{\beta}
$$


is admissible in BCK if and only if the corresponding quasi-identity

$$
\alpha_{1}=1 \& \cdots \& \alpha_{n}=1 \quad \longrightarrow \quad \beta=1
$$

holds in the free BCK-algebra $\mathbf{F}(X)$ on a countable set of generators $X$. If the rule is derivable, the corresponding quasi-identity holds in every BCK-algebra. So, admissible but nonderivable rules describe certain properties of the free BCK-algebra that are not shared by all BCK-algebras. We will exhibit one such property here.

By a Heyting chain we mean a BCK-algebra $\mathbf{H}$ such that $H$ is linearly ordered by the natural BCK ordering, and for all $a, b \in H$ we have $a \rightarrow b=b$ unless $a \leq b$. If a Heyting chain $\mathbf{H}$ is finite, it is often called $\mathbf{H}_{n}$ with $n>0$ being the cardinality of $H$. The next lemma is obvious.

Lemma 5.1 Let $\mathbf{H}$ be a Heyting chain such that $|H|>2$. Then $\mathbf{H}_{3}$ is a subalgebra of $\mathbf{H}$.

Theorem 4.2, phrased algebraically, says that for any $s, t \in F(X)$ we have

$$
(s \rightarrow t) \rightarrow t=1 \quad \text { implies } \quad(t \rightarrow s) \rightarrow s=1 .
$$

The result below is a corollary.

Theorem 5.2 No Heyting chain $\mathbf{H}$ is a subalgebra of $\mathbf{F}(X)$.

Proof By Lemma 5.1 it suffices to prove that $\mathbf{H}_{3}$ is not a subalgebra of $\mathbf{F}(X)$. Suppose that $\mathbf{H}_{3} \subseteq \mathbf{F}(X)$. Then there are terms $s, t \in F(X)$ with $1>s>t$ such that $s \rightarrow t=t$ and $t \rightarrow s=1$. Thus, $(s \rightarrow t) \rightarrow t=1$ but $(t \rightarrow s) \rightarrow s=1 \rightarrow s=s \neq 1$, contradicting Theorem 4.2.

\section{References}

[1] Blok, W. J., and D. Pigozzi, Algebraizable Logics, vol. 77 of Memoirs of the American Mathematical Society, Providence, 1989. MR 0973361. DOI 10.1090/memo/0396. 198

[2] Cintula, P., and G. Metcalfe, "Structural completeness in fuzzy logics," Notre Dame Journal of Formal Logic, vol. 50 (2009), pp. 153-182. MR 2535582. DOI 10.1215/00294527-2009-004. 197

[3] Curry, H. B., R. Hindley, and J. P. Seldin, Combinatory Logic, II, North Holland, Amsterdam, 1972.

[4] Iseki, K., "An algebra related with a propositional calculus," Proceedings of the Japan Academy, vol. 42 (1966), pp. 26-29. MR 0202571. 198

[5] Kowalski, T., "Self-implications in BCI," Notre Dame Journal of Formal Logic, vol. 49 (2008), pp. 295-305. MR 2428556. DOI 10.1215/00294527-2008-013. 199

[6] Olson, J. S., J. G. Raftery, and C. J. van Alten, "Structural completeness in substructural logics," Logic Journal of the IGPL, vol. 16 (2008), pp. 455-495. MR 2453364. DOI 10.1093/jigpal/jzn014. 197, 198

[7] Pogorzelski, W. A., "Structural completeness of the propositional calculus," Bulletin de l'Académie polonaise des sciences, vol. 19 (1971), pp. 349-351. MR 0302398. 197

\section{Acknowledgment}

The author is grateful to an anonymous referee for suggestions that helped to correct a few silly mistakes and led to a substantially improved presentation. 
Department of Mathematics and Statistics La Trobe University

Bundoora, 3086 Victoria

Australia

t.kowalski@latrobe.edu.au

http://tomasz-kowalski.Itumathstats.com/ 